\title{
What's HITECH About CRS-HIPEC?
}

\author{
Sweekar Kudpi Shenoy ${ }^{1}$, Eeshwar M $V^{2 *}$, Ramya Kamlekar ${ }^{3}$, Manjunath Prabhu ${ }^{4}$, Shreya Das \\ Adhikari $^{5}$, Shweta Sinha ${ }^{6}$, Yogesh K Gaude \\ 1,2,3,4,5,6,7 Kasturba Medical College, Manipal, India
}

\begin{abstract}
Hyperthermic intraperitoneal chemotherapy (HIPEC) is a technique, which delivers heated chemotherapeutic agents into the peritoneal cavity. It has been shown to improve prognosis and survival rate in patients of colorectal, ovarian and gastric cancers with peritoneal metastasis. A 57-year-old man diagnosed with primary colorectal carcinoma and pseudomyxoma peritonei was posted for HIPEC surgery. General anaesthesia with epidural analgesia (pain management) and invasive lines (arterial, central) for managing fluid shifts were used. Stroke volume variation was measured throughout the 19-hour procedure for goal directed fluid therapy (GDT) and to maintain urine output. Temperature monitoring with deliberate cooling measures were used during the period of HIPEC along with serial arterial blood gas analysis (ABG).
\end{abstract}

Keywords: hyperthermic intraperitoneal chemotherapy, HIPEC, anaesthetic management, urine output, stroke volume variation

\section{Introduction}

Peritoneal metastasis has been considered to be an incurable palliative condition until Dr Paul Sugarbaker introduced the concept of loco regional heated chemotherapeutic drugs. ${ }^{1}$ Once combined with cytoreductive surgery (CRS), HIPEC is shown to improve the 5-year survival in patients with incurable peritoneal metastasis. ${ }^{2}$ This procedure however is associated with major fluid shifts, fluctuations in temperature, extensive blood loss, major haemodynamic changes, electrolyte imbalances and coagulopathies. These issues present a major challenge to the anaesthesiologist.

*Correspondence: Eeshwar M V

E mail: eeshwar.kmc@gmail.com

iD https://orcid.org/0000-0003-4138-7814

Received: 17/10/2020

Accepted: 13/8/2021

DOI: https://doi.org/10.4038/slja.v29i2.8698

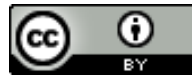

\section{Case Report}

A 57-year-old male with pre-existing controlled hypertension presented with complaints of abdominal distension for 7 months. $\mathrm{He}$ is a known patient with retroviral disease. His preoperative room air saturation was 95\%, respiratory rate was $14 / \mathrm{min}$, blood pressure was $110 / 80 \mathrm{mmHg}$ and pulse rate was $81 / \mathrm{min}$. Preoperative spirometry showed restrictive lung disease. His diffusion lung capacity for carbon monoxide (DLCO) levels was less than $55 \%$ of predicted. Consent for high risk surgery was obtained. A backup ventilator in the post anaesthesia care unit (PACU) was preserved in the view of the major surgery and underlying comorbid illnesses. Adequate blood and blood products were arranged preoperatively. RT PCR test was done to rule out COVID-19 preoperatively and the patient was tested negative. Cold intravenous (IV) fluids and ice packs were kept ready to manage the hyperthermia induced during the period of HIPEC (Figure 1).

On the morning of the surgery, standard monitors (ECG, non-invasive blood pressure, pulse oximeter) were attached to the patient. 
Two wide bore IV lines were secured inside the operating room (OR).

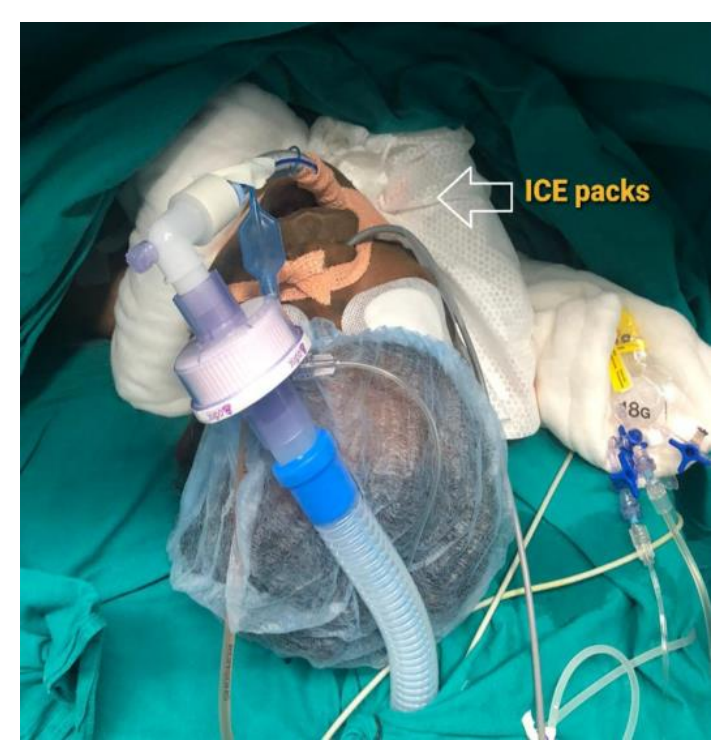

Figure 1. Placement of icepacks to manage hyperthermia

Epidural catheter was secured at the thoracic level T6 under sterile aseptic precautions. An arterial line was secured for continuous monitoring of blood pressure and connected to a FloTrac cardiac output (CO) monitor and his baseline stroke volume variation (SVV), CO were charted.

General anaesthesia was induced using IV propofol and paralysis was achieved with IV vecuronium and the patient was intubated using a cuffed endotracheal tube. Post intubation right internal jugular venous cannulation was performed using ultrasound. Epidural morphine and IV fentanyl and paracetamol were used for analgesia. Epidural infusion of local anaesthetic was used depending upon the haemodynamic status intraoperatively. Fluids (Ringer's lactate / Plasmalyte) were administered targeting a SVV less than $12 \%$ and a urine output of $1 \mathrm{~mL} / \mathrm{kg} / \mathrm{h}$ till the HIPEC was initiated. Blood loss was corrected once the maximum allowable blood loss was reached. The patient required two pints of packed cells and one fresh frozen plasma. The patient's body temperature was maintained at 33 to $34^{\circ} \mathrm{C}$ using fluid warmers and forced air blowers. About 5 liters of ascitic fluid was tapped that resulted in hypotension and IV noradrenaline was started at this time. IV $20 \%$ albumin was administered intraoperatively.

HIPEC was initiated after a 14-hour resection time of the primary tumor. A special machine is used to administer this type of chemotherapy (Figure 2). Adriamycin and Mitomycin were the drugs used during this phase. Four tubes were placed inside the peritoneal cavity and the agents were administered in $1.5 \%$ dextrose. (Figure 3 ) The temperature during this time rose to a maximum of $37^{\circ} \mathrm{C}$. Cold IV fluids $\left(18^{\circ} \mathrm{C}\right)$ and ice packs $\left(10^{\circ} \mathrm{C}\right)$ were used to reduce the temperature.

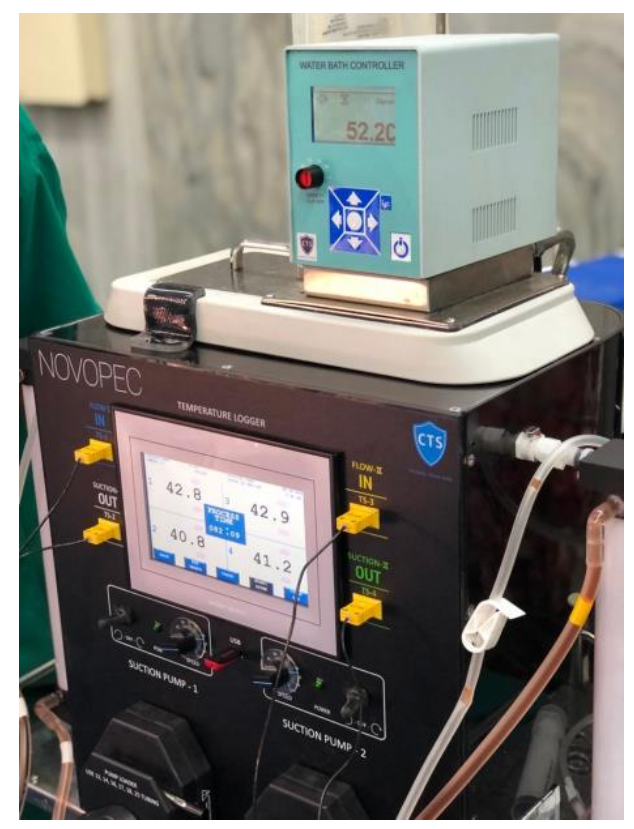

Figure 2. HIPEC chemotherapy machine

Urine output was maintained at $2-3 \mathrm{~mL} / \mathrm{kg} / \mathrm{h}$ during this phase. ABG's were done every 30 min (lactates and electrolytes) during the HIPEC phase, which lasted for $90 \mathrm{~min}$. Lactate rose from a baseline value of $13 \mathrm{mg} / \mathrm{dL}$ to $33 \mathrm{mg} / \mathrm{dL}$. Adequate perfusion was maintained with administration of fluid boluses and noradrenaline. Coagulation profile (PT, APTT and INR) was sent 30 minutes after the initiation 
of HIPEC. Blood glucose values were monitored at 30-minute intervals in view of risk of systemic absorption of $1.5 \%$ dextrose. Haemodynamics remained stable throughout this phase.

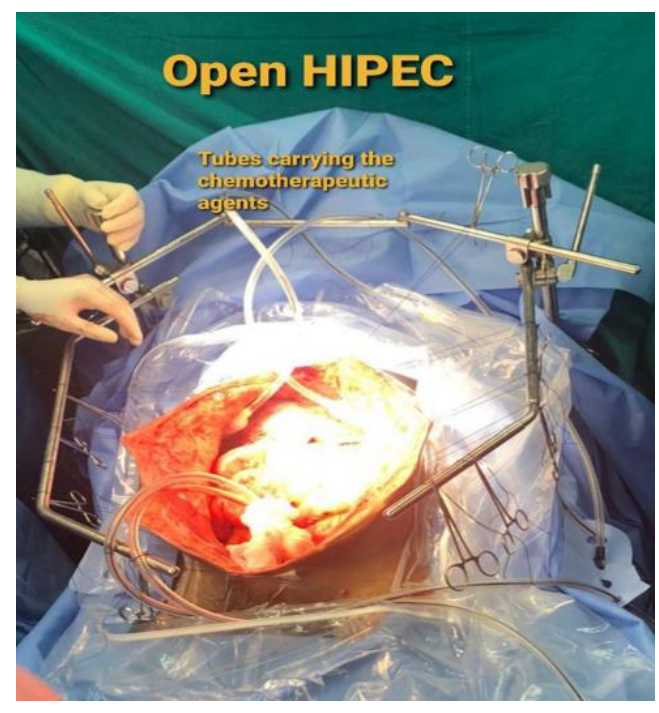

Figure 3. Open HIPEC procedure

The temperatures fell to $34^{\circ} \mathrm{C}$ after the HIPEC phase and the surgeon completed the anastomoses. The patient was shifted to the PACU for planned mechanical ventilation. In the PACU, X-ray and thromboelastrogram (TEG) were done immediately. Epidural infusion of local anaesthetics was initiated. Warming of the patient was continued. The patient was ventilated for 8 hours post-operatively and was gradually weaned of the ventilator along with tapering of noradrenaline. Post extubation, incentive spirometry and physiotherapy were initiated and the patient was shifted to the ward on post-op day 3 .

\section{Discussion}

HIPEC is a heated chemotherapy treatment delivered intra-abdominally during surgery. ${ }^{2}$ Adequate cytoreduction must be done prior to starting this chemotherapy as the drugs penetrate tissues only up to a depth of $5 \mathrm{~mm} .{ }^{1}$ After cytoreduction, a closed, semi-open or open abdomen technique may be used to perform HIPEC. ${ }^{3}$ Infusion catheters are inserted into the abdomen and a heated chemotherapy solution is circulated in the abdomen for 90-120 minutes using a heating circuit and roller pump. ${ }^{4}$ Temperatures of 41-43 degrees Celsius for 90 minutes result in inhibition of RNA synthesis and mitosis arrest in malignant cells. It results in an acidic environment for the tumour cells and activates lysosomal enzymes which accelerates death of the tumour cells. ${ }^{1}$

Chemotherapy can also be administered by a technique known as pressurized intraperitoneal aerosolized chemotherapy (PIPAC). This is done when a minimally invasive surgical approach (laparoscopy) is used. A nebulizer is connected to a high-pressure injector and a therapeutic capno-peritoneum is created. ${ }^{5}$ During such procedures, there is a risk of aerosolization and occupational exposure to the theatre personnel. N95 masks with a tight seal are recommended during this procedure. The nebulizer and the pressurized injector should be operated from outside the theatre using a remote control. The patient also must be monitored remotely. Risk of exposure to chemotherapeutic agents resulting in hair loss, headache, acute irritation, hypersensitivity and congenital malformations in fetus of pregnant women is a possibility. ${ }^{5}$ In the cytoreductive phase of the procedure, hypothermia may occur which may be managed with drapes, warming blankets, fluid warmers and body warmers. ${ }^{3}$ Before commencing HIPEC phase, it is essential to switch to cold IV fluids and remove all warming devices in anticipation of the hyperthermia. ${ }^{4}$ Hyperthermia may increase systemic oxygen demand and result in metabolic acidosis as well as rising end tidal $\mathrm{CO}_{2}$ levels. ${ }^{2}$

Cytoreduction is associated with significant third space losses, major fluid shifts and bleeding. ${ }^{4} \mathrm{~A}$ goal directed approach to fluid therapy is preferred over a liberal approach to minimize fluid overload. ${ }^{3}$ Goal directed therapy assesses patient's response to fluids from hemodynamic changes as per frank starling curve. ${ }^{6}$ In our patient we used FloTrac to measure the SVV. A fluid responsive patient will demonstrate an 
increase in SVV by $10-15 \%$ after a fluid challenge. ${ }^{7}$ This uses pulse contour analysis to calculate cardiac output. Predictors of fluid responsiveness include high pulse pressure variation and stroke volume variation. During the HIPEC phase the saline enriched chemotherapeutic drugs may increase the intraabdominal pressure, decrease venous return and decrease the $\mathrm{CO} .{ }^{1}$ Urine output monitoring is essential during fluid management. A urine output of $0.5 \mathrm{~mL} / \mathrm{kg} / \mathrm{hr}$ is required during the CRS phase and $4 \mathrm{~mL} / \mathrm{kg} / \mathrm{hr}$ is essential during the HIPEC phase of surgery. ${ }^{3}$ It is important to consider the nephrotoxic effects of chemotherapeutic agents and hence an average diuresis of $500 \mathrm{~mL} / \mathrm{hr}$ is needed during the HIPEC phase. $^{4}$ Dextrose containing peritoneal carrier solution used for HIPEC may cause hyperglycemia in these patients and hence frequent monitoring of blood glucose is essential. ${ }^{1}$ Consequences may include lactic acidosis and dilutional hyponatremia. Frequent $\mathrm{ABG}$ monitoring is needed to find and correct any abnormalities. ${ }^{1}$

During CRS, dilutional coagulopathy may occur. Hyperthermia may also result in coagulopathies. ${ }^{3}$ Coagulation studies can be routinely done to assess the same. In our case TEG and PT/INR with APTT were performed to assess for any coagulopathies. Red cells and plasma were transfused as needed.

This is a very painful procedure, and an epidural is essential for post-operative pain relief. ${ }^{2}$ Multimodal analgesia combining local anaesthetics and opioids via thoracic epidural reduce post-operative hospital stay, chances of post-operative ileus and impaired ventilation. ${ }^{1}$

\section{Conclusion}

CRS with HIPEC is a complex procedure which results in extreme fluid shifts, blood loss and temperature changes. A goal directed approach to fluid management can then be applied by the anaesthetist ensuring that the patients remain euvolaemic. Good coordination with the surgeons and anaesthetist is essential for a satisfactory perioperative outcome.

\section{References}

1. Gupta N, Kumar V, Garg R, Bharti SJ, Mishra S, Bhatnagar S. Anesthetic implications in hyperthermic intraperitoneal chemotherapy. $J$ Anaesthesiol Clin Pharmacol 2019; 35:3-11

2. Kearsley R, Egan S, McCaul C. Anaesthesia for Cytoreductive Surgery with Hyperthermic Intraperitoneal Chemotherapy (HIPEC). ATOTW 3792018

3. Lewis A, Dabo-Trubelja A. Anesthesia for cytoreductive surgery with heated intraperitoneal chemotherapy. Up to Date 2020.

4. Chawla A, Zhu C-C, Backer G, O'Gara J, Fong ZV,Deng $\mathrm{H}$, Bao X, Cusack J. Perioperative Management of Patients Undergoing CRS and HIPEC. Clin Surg. 2020; 5: 2788.

5. Solanki SL, Mukherjee S, Agarwal V, et al. Society of Onco-Anaesthesia and Perioperative Care consensus guidelines for perioperative management of patients for cytoreductive surgery and hyperthermic intraperitoneal chemotherapy (CRS-HIPEC). Indian Journal of Anaesthesia. 2019 Dec; 63(12):972.

6. Kendrick JB, Kaye AD, Tong Y, Belani K, et al. Goal-directed fluid therapy in the perioperative setting. Journal of anaesthesiology, clinical pharmacology. 2019 Apr; 35(Suppl 1): S29.

7. Bennett VA, Cecconi M. Perioperative fluid management: From physiology to improving clinical outcomes. Indian J Anaesth 2017; 61:614-21. 